\title{
The Amino Acid Pool in Oncology: Plastic Substrates, Specific Regulators of Metabolism and Markers
}

\section{Karavay $\mathbf{P}^{1}$, Karavay $\mathbf{N}^{2}$ and Nefyodov $\mathbf{L}^{3 *}$}

${ }^{1}$ Department of Oncology University of Grodno State Medical, Grodno, Belarus

2 Department of Hyperbaric Oxygenation of Grodno Regional Hospital, Grodno, Belarus

${ }^{3}$ Department of Biochemistry University of Grodno State, Grodno, Belarus

\begin{abstract}
On the basis of experimental data we suggest that discovered differences in certain amino acids concentrations in blood plasma, red blood cells and tumors are criteria in early diagnostic of primary cancerous growth as well as, in estimation of the effectiveness of specific cancer treatment. In clinical studies (1,495 patients with cancer of mammary gland, lungs, prostate, ovaries, bladder or digestive tract) biological fluids and tumors of cancer patients significant changes of physiological concentrations of amino acids real or indirectly regulate processes of the antitumor response, oncogenesis, immunogenesis and apoptosis were shown. Our strategy of application of amino acids as medicinal preparations includes a directional effect on the functional and metabolic relationships which change in specific pathology through the effect on the regulatory mechanisms of intermediate metabolic reactions, limiting stages of metabolic flows, utilization of energy substrates and transport systems restricting the processes of amino acids pool formation. The creation methodology of pathogenetic compositions of amino acids and their derivatives on the basis of their physiological concentration for practical application in oncology of their regulatory effects was discussed.
\end{abstract}

Keywords: Free amino acids; Regulatory effects; Oncology

\section{Introduction}

Amino acids and their derivatives are mostly universal natural regulators and endogenous modifiers of biological reactions. However, the numerous biological properties of these compounds as drugs were used for correction of deficiencies or realization of pharmacological and immediate metabolic effects, disregarding a regulatory action. Amino acid profile indexes that allow early detection of diseases that would provide time for intervention before irreversible damage occurred are being created. Thus, amino acid profiles represent biomarkers for diseases or deviations from a normal state of health. Our array technology will play an important role in metabolomics in biomarker discovery, clinical medicine, including cancer as well as, in other stages of drug discovery and development (for example, target discovery, mechanism of action or predicting toxicity) [1-6]. Changes in amino acid fund of liquids and tissues of patients specifically characterized cancer illness. Correction of the intermediate metabolic changes in cancer can be reached at use of separate amino acids or their combination $[7,8]$. To date, there are main levels of exploitation of biochemical (metabolic) properties of amino acids and their derivatives.

\section{In clinical practice}

- The use of amino acids or multicomponent mixtures of amino acids (mainly, essential elements combined with vitamins and trace elements) for replacement therapy or shortfall of essential nutrients and proteins.

- The use of drugs on the basis of individual amino acids or their compositions developed based on the additive functional and metabolic action, in which the "exploited" pharmacological activity (effects of activation of redox processes, reactions of energy metabolism and neutralization of xenobiotics compounds) of this class (VI Ordinary General Assembly Society of Biochemistry of Amino acids and their derivatives in biology and medicine [9-11].

However, the use of certain levels of L-amino acids or their compositions or shortfall implements direct pharmacological effects that practically ignored their regulatory effects on metabolic processes and key metabolic reactions.

To understand the metabolic processes and vital functions of the regulatory effect of amino acids, this manifests itself under natural or near concentrations of these compounds in body fluids and tissues. It is obvious that the effective use of L-amino acids or their derivatives for metabolic correction and directional changes in metabolism in pathological or extreme conditions limited accumulation of information about theme mechanisms of regulatory effects of the compounds tested at concentrations comparable to their physiological (endogenous) level [1-10].

\section{Materials and Methods}

\section{Our development methodology is based on}

- The study of physiological concentrations of free amino acids, their derivatives, precursors and metabolites, as well as, biochemical marker parameters in healthy donors and patients with various pathologies.

- Creating a unified database for the studied parameters, construction of empirical mathematical model consisting of pathogenic markers specific pathology and amino acid profiles.

- Specialized development of new formulations of the compositions of infusion solutions of amino acids and their

${ }^{*}$ Corresponding author: Nefyodov L, Department of Biochemistry, University of Grodno State, Belarus 22, Ozeshko Str, Grodno, 230023, Tel: +375 (29) 6376 982; E-mail: nefyodov@mail.ru

Received October 13, 2015; Accepted December 29, 2016; Published January 06, 2017

Citation: Karavay P, Karavay N, Nefyodov L (2017) The Amino Acid Pool in Oncology: Plastic Substrates, Specific Regulators of Metabolism and Markers. Pat Care 3: 126 . doi: $10.4172 / 2573-4598.1000126$

Copyright: (c) 2017 Karavay P, et al. This is an open-access article distributed under the terms of the Creative Commons Attribution License, which permits unrestricted use, distribution, and reproduction in any medium, provided the original author and source are credited. 
derivatives [1-10]. The results and our concept is based on research of formation of free amino acids fund in biological liquids and tissues of 1,495 of patients with cancer of mammary gland, lungs, prostate, ovaries, bladder or digestive tract.

\section{Results and Discussion}

Numerous results of determination of amino acids and their derivatives in human body fluids and tissues allowed systematization of the accumulated data and identified areas for exploitation of their metabolic effects, primarily, in laboratory diagnostics and application in clinical practice as drugs $[4,5]$. In view of the fact that the free amino acids are represented by a wide range of related chemical structure and metabolic transformations of compounds that forms in the body fluids and tissues, amino acid found proved that quantification of their pool contributes to the diagnosis of various diseases, including hepatobiliary pathology, cardiovascular and immune systems, oncological causes, cerebrovascular pathology, alcoholism and diabetes [1-6]. It turned out that the vast majority of the diagnostic values of the group have shifts in the level of functional and metabolic related amino acids and their derivatives and as such no specific changes in the concentrations of individual compounds of this class is observed. Metabolic related amino acids and their derivatives had the nature of the amino acid profiles of the body fluids and tissues of animals and humans when compared with the use of multivariate analysis and mathematical modeling. At the same time, it was convincingly demonstrated that the removal or correction of the intermediate metabolic changes can be achieved using individual amino acids and their derivatives or a combination of them as universal natural bioregulators - compounds that has direct effect on the mechanisms of cellular metabolism in physiological concentrations [1-3]. To date, there are evidences of the importance of not only amino acids as building blocks for protein synthesis, but regulators of gene expression at the level of mRNA translation by mTOR-dependent mechanism, signaling molecules and bio- logical response modifiers, as well as, precursors of a wide range of bioregulators, which play a key role in the integration of major metabolic fluxes [12-16].

Based on the positions of metabolomics, the amino acid pool of biological fluids and tissues found free amino acids evaluated as a single information unit, which is a kind of "chemical projection" of the genome, proteome realized through this approach not only develops ideas about the pool of amino acids as a dynamical system generated receipt of them from outside, but also due to endogenous synthesis, transport, degradation and excretion and allows the identification of "key points" intermediate metabolic equilibrium shift that may reflect ratios at the individual levels of endogenous amino acids and related species (metabolic related) compounds. We were the first to demonstrate that endogenous levels of free amino acids in fluids and tissues are the most important integral indicators and regulators of metabolism. This enables us to prove the use of individual amino acids or their combinations for guided correction of metabolism with specific human diseases and significantly expand the area of practical application of these compounds as blood substitutes. Under regulator effects of amino acids their effect on the biochemical processes and vital functions, which is manifested in natural (endogenous) or close to natural concentrations of these compounds is understood.

A regulatory effect after introduction of amino acids can be achieved by using either individual amino acids, or a combination of their small sets. The structure of these compositions in specific ratios may include almost any amino acids, their structural analogs or derivatives with a known mechanism of action. The trend developed is notable for the fund of amino acids in biological fluids and tissues are assessed as a single information unit and while analyzing it, it is still possible to estimate the segments which disrupt the metabolic balance (changes in the metabolic flows ratio and their balance shifts).

Our proposed methodology for developing new formulations multicomponent infusion solutions based on amino acids and related compounds for the correction occurring in various diseases of the metabolic imbalance is based on the application of research results regularities of formation of amino acid found in biological fluids and tissues in various pathological conditions. The composition and quantity of highly purified amino acids in these infusion solutions should be determined primarily by their physiological (regulatory) concentrations, which distinguishes them from the traditionally used amino acid solutions for parenteral nutrition, where the content of their components is calculated based on the daily needs of the human body in them without due consideration of regulatory actions administered to compounds. Separate amino acids or their compositions in oncological practice should be applied according to their physiological concentrations and change of structure of amino acids fund of patients. Amino acids with anti-carcinogenic effects may be leucine, tryptophane and taurine.

\section{Conclusion}

The methodology of development of new compoundings of multicomponent infusion solutions offered by us on the basis of amino acids and the related connections is intended for correction of the metabolic imbalance arising at various diseases based on application of results of research regularities of formation of amino acid found in biological liquids and tissues of individuals at most various pathological states (more than 9000 surveyed) [1-3]. Separate amino acids or their compositions in oncological practice should be applied according to their physiological concentrations and change of structure of amino acids fund of patients. As a result of numerous experimental and clinical studies, we proved that diagnostically significant in oncology and "anticarcinogenic" are amino acids leucine, tryptophan and taurine. The new methodology of development of minicomponents infusion solutions offered by us on the basis of amino acids intended for correction of the metabolic imbalance arising at various localization and stages of the malignant growth is based on results of research of regularities of formation of amino-acid fund in biological liquids and tissues. The ratios of the individual components and their quantity in such mixtures should comply with their physiological (endogenous) concentrations in the normal blood plasma and tissues [17-21].

\section{References}

1. Nefyodov L (1999) Taurine (biochemistry, pharmacology, medical application). Grodno RIPH 145

2. Amino Acids and their derivatives (1996) Grodno 125.

3. Nefyodov LI, Karavay PA, Karavay NL (2014) Regulatory action of free amino acids and development on the basis of highly of substances infusion solutions with pathogenetic deterministic composition. Laboratory Diagnosis Eastern Europe 3: $111-115$

4. Holden JT (1962) Amino acid pools. Elsevier, Amsterdam, p: 815

5. Lubec G (1990) Amino Acids: Chemistry, Biology, Medicine. Escom, New York 1196.

6. Nefyodov LI (2010) The results of biochemical research and development of nitrogen-containing compounds of natural origin: Methodology of exploitation of biological properties as universal natural regulators of metabolism and drugs. Research Laboratory of biochemistry of biologically active substances.

7. Nefyodov LI (1996) Amino acids and their derivatives in tumour tissue from 
Citation: Karavay P, Karavay N, Nefyodov L (2017) The Amino Acid Pool in Oncology: Plastic Substrates, Specific Regulators of Metabolism and Markers. J Pat Care 3: 126. doi: 10.4172/2573-4598.1000126

Page 3 of 3

patients with breast cancer treated with Ukrain: Part VI. Drugs Exp Clin Res 22: 155-157.

8. Biological activity and transport of drugs (1999) Grodno, p: 189

9. VI Ordinary general assembly society of biochemistry of belarus (2000) Grodno, p: 225.

10. Nefyodov (2001) Amino acids and their derivatives in biology and medicine. Grodno, p: 124.

11. Nefyodov LI (2001) Target-oriented regulation of metabolic equilibrium by amino acids and strategy of their application as drugs with directional effects. Torun, p: 327.

12. Ihata Y, Miyagi E, Numazaki R, Muramatsu T, Imaizumi A et al. (2014) Amino acid profile index for early detection of endometrial cancer: Verification as a novel diagnostic marker. J Clin Oncol 19: 364-372.

13. Fafournoux $P$, Bruhat $A$, Jousse $C$ (2000) Amino acid regulation of gene expression. BioChem J 351: 1-12.

14. Meijer A (2003) Amino acids as regulators and components of nonproteinogenic pathways. J Nutr 6: 2057S-2062S.
15. Bruhat A, Cherasse $Y$, Chaveroux $C$, Maurin AC, Jousse $C$ et al. (2009) Amino acids as reg- ulators of gene expression in mammals: Molecular mechanisms. Biofactors 35: 249-257.

16. Ananieva $E$ (2015) Targeting amino acid metabolism in cancer growth and antitumor immune response. World J Biol Chem 6: 281-289.

17. Hong Ma, Hasim A, Mamtimin B, Kong B, Zhang HP, et al. (2014) Plasma free amino acid profiling of esophageal cancer using high-performance liquid chromatography spectroscopy. World J Gastroenterol 20: 8653-8659.

18. Karavay PA (2016) Amino acids in metabolomics: Perspective for the use of regulatory effects of fee amino acids in the creation on their basis of infusion solutions.

19. http://www.ommegaonline.org/journal-details/International-Journal-ofHematology-and-Therapy/44

20. Karavay A, Karavay P, Kaliada T, Nefyodov L (2016) Metabolic comfort in oncology and free amino acids: Perspectives for the use of their regulatory actions in physiological concentrations.

21. Karavay A, Karavay P, Koliada T, Nefyodov L (2016) Academic Web Journal of Medical Science 1: 2545-4986. 